\title{
Le Musée européen des copies de Charles Blanc comme « pendant » du Louvre
}

Charles Blanc's Musée européen des copies as a "pendant" to the Louvre

\section{Elisa Rodríguez Castresana}

\section{OpenEdition} Journals

Édition électronique

URL : http://journals.openedition.org/cel/731

DOI : $10.4000 /$ cel. 731

ISSN : 2262-208X

Éditeur

École du Louvre

Référence électronique

Elisa Rodríguez Castresana, «Le Musée européen des copies de Charles Blanc comme «pendant » du Louvre », Les Cahiers de l'École du Louvre [En ligne], 11 | 2017, mis en ligne le 26 octobre 2017, consulté le 17 septembre 2019. URL : http://journals.openedition.org/cel/731; DOI : 10.4000/cel.731

Ce document a été généré automatiquement le 17 septembre 2019

\section{(c) (1) $\odot$}

Les Cahiers de l'École du Louvre sont mis à disposition selon les termes de la licence Creative Commons Attribution - Pas d'Utilisation Commerciale - Pas de Modification 4.0 International. 


\title{
Le Musée européen des copies de Charles Blanc comme " pendant » du Louvre
}

Charles Blanc's Musée européen des copies as a "pendant" to the Louvre

\author{
Elisa Rodríguez Castresana
}

1 Notre objectif est d'étudier le Musée européen des copies, ouvert en 1873 sous l'impulsion du directeur de l'administration des Beaux-Arts Charles Blanc, et les modalités de son appropriation du Louvre. Cet article sera l'occasion d'analyser la construction idéologique très complexe qui préside à son ouverture, ainsi que le rôle clé de modèle joué par le Louvre dans cette construction.

\section{Le Musée européen des copies et son créateur}

2 Le principal promoteur du Musée européen des copies a été Charles Blanc, un grand historien de l'art qui occupe le poste de directeur de l'administration des Beaux-Arts à deux reprises : une première fois entre 1848 et 1851 , puis entre 1871 et 1873. Blanc a créé la Gazette des Beaux-Arts et publié de très nombreux textes sur l'art et son histoire, dont l'un est un vrai best-seller, La Grammaire des Arts $d u$ dessin ${ }^{1}$. Il occupe la première chaire française dédiée à l'esthétique et à l'histoire de l'art, créée spécialement pour lui au Collège de France en 1878.

3 Le Musée européen des copies est inauguré en avril $1873^{2}$. Cependant, Charles Blanc a commencé à imaginer ce musée presque trente ans auparavant, lorsqu'il était directeur des Beaux-Arts dans le gouvernement de la Deuxième République, c'est-à-dire entre 1848 et 1851. Si nous lisons le rapport adressé par Blanc en 1871 au ministre de l'Instruction publique, des Cultes et des Beaux-Arts Jules Simon, nous constatons que l'origine de son projet remonte encore plus loin, à l'époque où Adolphe Thiers était encore ministre de l'Intérieur, en $1832^{3}$. Mais s'il est vrai qu'Adolphe Thiers partageait avec Charles Blanc un même intérêt pour les copies, les objectifs du projet de musée, 
que le ministre de Louis-Philippe mit en œuvre à l'École des beaux-arts, diffèrent de ceux du Musée européen des copies. Nous verrons toutefois que la figure de Thiers a joué un rôle essentiel dans la création puis la disparition du musée.

4 Pour comprendre le projet de Charles Blanc, il suffit de lire le rapport qu'il adressa à Jules Simon le 26 octobre 1871 :

[...] je veux dire le projet de fonder en France un Musée Universel, renfermant des copies excellentes, des moulages parfaits, et des épreuves de choix, de tout ce qu'il y a de beau dans le monde entier. L'histoire des Arts se déroulerait ainsi dans une galerie magnifique et sans pareille, dont les parois seraient couvertes de bas-reliefs antiques et modernes depuis les Égyptiens jusqu'à Puget, depuis Phidias jusqu'à l'Algarde. [...] Les chambres du Musée Universel s'ouvriraient, ici par les portes d'Andrea Pisano ; là par celles de Ghiberti, [...] Dans les salles de peinture, seraient placées les stances de Raphaël [...] Sur le pavement, seraient reproduits les Graffiti de Beccafumi à Sienne ; les mosaïques pompéïennes [sic] de la Bataille d'Arbelles [...] Des estampes, des photographies, la collection de la chalcographie du Louvre et des épreuves d'après les cuivres étrangers, tapisseraient les vestibules, les paliers, sans préjudice de quelques grandes tapisseries et de quelques vases de Sèvres [... $]^{4}$

Le Musée des copies devait être un musée universel, propre à renfermer les copies des plus grands chefs-d'œuvre de l'histoire de l'art. Dans les faits, Charles Blanc se vit forcé de revoir son concept à la baisse. À son ouverture, en avril 1873, le musée ne comprenait plus qu'une centaine de copies exécutées d'après les chefs-d'œuvre de la peinture européenne.

Avec la création du Musée européen des copies, Charles Blanc poursuivait quatre objectifs principaux. Le premier était de rationaliser le système d'envois du prix de Rome et de venir en aide aux artistes qui pâtissaient de la difficile situation économique de la France après la guerre contre la Prusse (1870-1871). Deuxièmement, Blanc entendait promouvoir la formation artistique aussi bien des artistes eux-mêmes que du grand public. Le troisième enjeu du projet était de conserver l'image des grands chefsd'œuvre de l'histoire de la peinture. Enfin, Charles Blanc souhaitait promouvoir la peinture d'histoire.

Les trois derniers objectifs fixés par Charles Blanc reposaient sur l'idée d'une relation étroite entre le Musée des copies et le musée du Louvre, qui constituait depuis longtemps le musée d'art de référence en France.

La création du Musée européen des copies, se positionnant en quelque sorte en miroir du musée du Louvre, doit être également considérée dans le contexte du travail effectué par Charles Blanc à la tête de la direction des Beaux-Arts. Il ne faut pas oublier que pendant les deux directorats de Blanc, le Louvre ainsi que le Salon des artistes vivants connaissent d'importantes mutations.

\section{La rationalisation du prix de Rome}

Le projet du Musée européen trouve son origine première dans le corpus des copies exécutées à titre d'envois par les pensionnaires de l'Académie de France à Rome ${ }^{5}$. Pendant leurs années de formation à Rome, les artistes pensionnaires - toutes disciplines confondues - doivent exécuter une copie d'après une grande œuvre du passé. L'exercice a été imaginé par le ministre Colbert sous Louis XIV. L'origine prestigieuse de cette politique d'État est souvent rappelée par Charles Blanc.

Conscient de l'importance de l'investissement consenti par l'État pour former des artistes à Rome, Blanc décide en 1871 de rationaliser les envois des pensionnaires. La 
collection du Musée européen est pensée comme un moyen de réunir ces envois, complétés chaque année par les nouveaux pensionnaires de l'Académie. Cependant, le nombre de copies obtenues par ce biais se révèle insuffisant pour remplir le Musée européen des copies en 1873, et Charles Blanc sait qu'il devra trouver d'autres moyens de constituer la collection.

\section{L'aide aux artistes}

De tous côtés, en effet, on nous signale chez les artistes réduits à la misère, par la cessation prolongée du travail, des projets d'émigration à l'étranger. Projets encouragés par les offres séduisantes qui leur sont faites, non seulement en Angleterre, en Russie, en Autriche, mais encore en Amérique ${ }^{6}$.

7 La situation des artistes après la guerre contre la Prusse est très précaire ; leur clientèle se raréfie. Charles Blanc envisage le Musée européen des copies comme un moyen de leur venir en aide, sans devoir acquérir des œuvres originales de moindre intérêt pour l'État. Si la commande de copies n'est pas une pratique nouvelle ${ }^{7}$, Charles Blanc ne s'adresse pas à des copistes professionnels - à cette époque, ce milieu recrutait surtout des femmes ${ }^{8}$ et plus rarement de jeunes artistes ou des peintres en difficulté. Charles Blanc considère que son musée doit permettre d'aider les artistes tout en contribuant à leur formation. Nous pouvons le lire dans un de ses articles sur la situation des BeauxArts avant le Salon de 1874 :

[...] mais le but principal d'une telle fondation était d'amener un certain nombre d'artistes éminents à se mettre en présence des grands maîtres, à se mesurer avec eux, à se retremper dans un commerce intime avec leurs œuvres. [...] L'intention du ministre des beaux-arts était de multiplier, en quelque sorte, les prix de Rome, en faisant pour une vingtaine de peintres choisis ce que fait chaque année le gouvernement pour trois ou quatre pensionnaires [...] $]^{9}$.

Pour Blanc, les artistes envoyés en Europe pour exécuter ces copies doivent jouir d'une réputation confirmée. Cette option vise à légitimer la qualité du futur musée auprès de son public potentiel. Blanc se place ainsi dans la continuité des pratiques académiques.

Cependant, si l'on en juge d'après quelques critiques publiées sur le musée à son ouverture en 1873, le choix des artistes n'a sans doute pas été aussi sélectif que promis. Le catalogue du musée rédigé par Louis Auvray nous communique la liste des peintres commandités par Charles Blanc ${ }^{10}$. Environ $40 \%$ de ces artistes n'ont jamais reçu de récompense, un chiffre très élevé eu égard à l'importance accordée par Blanc à la renommée des artistes comme fondement de la légitimité du musée.

En fait, le projet est mené avec une précipitation qui a nui à la qualité de son exécution. Charles Blanc est conscient de la fragilité de sa position politique, qui dépend de la présence d'Adolphe Thiers à la tête de la République. Ces deux hommes savent qu'une fois le territoire français libéré de l'armée prussienne, Thiers sera rapidement neutralisé par la faction royaliste de l'Assemblée nationale. Les commandes sont donc passées au plus vite, et souvent à des artistes de faible envergure. Prenons le cas de la Madone Sixtine de Raphaël. Le musée est inauguré en avril 1873, sans que la copie de ce tableau ne paraisse sur les cimaises, alors qu'il s'agit là d'un des plus importants chefsd'œuvre de l'histoire de l'art.

Cette anomalie, au premier regard anecdotique, montre bien la fragilité du projet de Charles Blanc: un projet qui devait toute sa survie à la domination politique d'Adolphe Thiers. L'engagement politique des principaux promoteurs du Musée européen des copies est un des éléments qui permettent d'expliquer son échec. 


\section{Relations entre le Musée européen des copies et le Louvre}

10 La question de la relation existant entre le Musée européen des copies et le musée du Louvre préoccupe déjà le public entre 1871 et 1874. En effet, en lisant la presse de l'époque, on remarque une certaine récurrence de la comparaison entre ces deux institutions. Cependant, nos recherches nous ont permis d'établir que les liens entre ces deux musées sont bien plus profonds, et tiennent non seulement à leurs collections respectives, mais aussi à leur construction idéologique.

\section{L'accrochage : transformation du Salon carré en 1848}

11 Charles Blanc dirige l'administration des Beaux-Arts pour la première fois en 1848 ; le musée du Louvre connaît un développement majeur cette année-là. Le nouveau directeur du musée, Philippe-Auguste Jeanron, décide de transformer le Louvre en lui donnant une nouvelle identité ${ }^{11}$. L'une des décisions les plus déterminantes pour la suite de l'histoire du musée parisien concerne le Salon des artistes vivants, dont Jeanron décide de ne plus accueillir son exposition annuelle au Louvre. Jusqu'en 1848, le Salon prend place au Salon carré, et pendant toute la durée de l'exposition, on retire à la vue les œuvres des maîtres anciens qui occupent les lieux le reste de l'année ${ }^{12}$. Malgré son succès auprès du grand public, aux yeux du nouveau directeur, le Salon ne constitue plus une priorité pour le Louvre.

\section{Un nouvel accrochage pour le Louvre}

En 1848 une page se tourne dans l'histoire du grand musée. La nouvelle direction décide de proposer un accrochage plus en accord avec le rôle pédagogique du musée. PhilippeAuguste Jeanron et Frédéric Villot imaginent ainsi un double accrochage: ils envisagent une exposition chronologique et par écoles, complétée par deux salles ordonnées suivant un critère différent ${ }^{13}$. La nouvelle présentation des œuvres au Louvre est perçue comme un tournantdans l'histoire du musée. En intégrant un critère chronologique dans la présentation, le Louvre assume son rôle pédagogique essentiel dans la diffusion de l'enseignement de l'histoire de l'art en France.

Le Salon carré et la Salle des sept cheminées deviennent des salles de chefs-d'œuvre, l'une consacrée aux écoles anciennes, l'autre à l'école française moderne. Nous nous concentrerons sur le dispositif mis en place au Salon carré, en raison de sa proximité avec le projet du Musée européen des copies. Le Salon carré constitue un espace à haute valeur symbolique. Au cours de l'histoire du palais, d'importantes célébrations ont eu lieu dans cet espace emblématique, telles que le mariage de Napoléon I avec l'archiduchesse Marie-Louise. Très probablement, en raison de sa puissance symbolique, mais aussi de son format inhabituel, Philippe-Auguste Jeanron décide de transformer cette salle d'exposition en salle des chefs-d'œuvre.

14 Comme Cecilia Hurley et François Mairesse l'expliquent dans leur article «In the shadow of the Tribuna ${ }^{14}$ ", c'est exactement à ce moment qu'un nombre appréciable d'institutions européennes décident de créer des salles de chefs-d'œuvre inspirées de la plus célèbre d'entre elles, la Tribuna des offices de Florence. Le nouveau dispositif mis 
en place au Salon carré sera autant admiré que décrié par la critique. Si l'intérêt du dispositif a été reconnu, le manque d'harmonie de l'accrochage, et la concurrence établie entre les différentes œuvres semblent poser un problème ${ }^{15}$.

L'introduction d'une salle exclusivement dédiée à l'exposition des chefs-d'œuvre rompt avec la relative monotonie de l'accrochage chronologique qui règne alors au Louvre. En choisissant d'inclure une salle de ce type, la nouvelle direction du musée du Louvre, appuyée par l'administration des Beaux-Arts, légitime son discours sur le rôle du musée. Le Louvre se positionne ainsi entre les deux conceptions du musée qui s'opposent à l'époque ${ }^{16}$ : le musée d'art et le musée d'histoire de l'art. Jeanron crée un musée complet, destiné aussi bien à l'étude de l'histoire de l'art qu'à la contemplation du beau absolu.

Nous pouvons nous demander quelle a été l'option choisie par Charles Blanc pour ordonner le Musée européen des copies. Blanc allie sa préoccupation pour la démocratisation de l'éducation artistique et son intérêt pour le dispositif de la salle des chefs-d'œuvre ; ces deux options d'accrochage, distinctes au sein du Louvre, fusionnent au Musée européen des copies. En fait, Charles Blanc décide d'appliquer l'accrochage chronologique et par écoles au dispositif de la salle des chefs-d'œuvre. La visée pédagogique de ce dispositif est encore soulignée par les propos de Charles Blanc luimême :

Ce que M. Thiers a fait pour lui-même, un gouvernement serait bien venu de le faire pour tous. Au lieu de ces interminables musées de peinture où abonde le médiocre, nous aurions des galeries réformées où l'on n'admettrait que l'excellent. Dans un autre Louvre, dans un Louvre cosmopolite, où figureraient tous les chefs-d'œuvre de sculpture et peinture qui sont dans le monde $[. . .]^{17}$.

Dans son texte consacré à la collection d'Adolphe Thiers - collection constituée en grande partie de copies - Blanc argumente en faveur de la constitution d'une collection réunissant uniquement les chefs-d'œuvre de l'histoire de l'art. Pour parvenir à ces fins, la copie et le moulage se révèlent des outils indispensables.

Nous pouvons ajouter un autre argument. Charles Blanc considère la salle et la collection de chefs-d'œuvre comme un dispositif pédagogique, car ce dernier concentre l'attention du visiteur. Au lieu de se perdre dans une multitude de salles, où l'œil non expert peine à démêler l'excellent du médiocre, le visiteur peut, aussi bien au Salon carré du Louvre qu'au Musée européen des copies, contempler un résumé de l'histoire de la peinture, illustrée par la copie de chefs-d'œuvre.

\section{La collection : une vision complète de l'histoire de l'art}

La collection du Musée européen des copies, nous l'avons vu, est uniquement composée de copies d'après les plus grands chefs-d'œuvre de l'histoire de la peinture européenne, à l'exception des chefs-d'œuvre exposés au Louvre.

Cette collection est donc ordonnée en fonction du Louvre. L'objectif de Charles Blanc est de pallier les lacunes de la collection du Louvre, et de proposer le panorama le plus complet possible de l'histoire de l'art et de la peinture. Cet objectif est perçu, et même mis en exergue par les critiques favorables à la création du musée. Nous le voyons très clairement dans cet argument développé par Louis Auvray :

[...] les artistes verraient annuellement s'augmenter la série des tableaux de maîtres que le Louvre ne possède pas et qu'ils viendraient étudier, et le peuple, qui ne peut voyager en touriste, aurait sous les yeux, classés par écoles, les chefs-d'œuvre 
disséminés dans les monuments et les plus riches galeries de l'Europe, groupés

chronologiquement dans ce Musée européen ${ }^{18}$. Le musée de Charles Blanc répond ainsi à une seule préoccupation : proposer une vision complète de l'histoire de la peinture à Paris, à travers la collection du Louvre d'une part, et celle du Musée européen des copies de l'autre. Les deux collections sont ainsi programmées comme complémentaires, et doivent être visitées l'une après l'autre pour donner au visiteur une idée complète de l'histoire de la peinture.

\section{La lutte pour l'enseignement artistique}

L'approche de Charles Blanc trahit une volonté de démocratiser l'art et son enseignement. L'objectif poursuivi par l'historien de l'art est de rapprocher des Français les œuvres capitales de l'histoire de la peinture. Au XIX $x^{e}$ siècle, l'enseignement de l'histoire de l'art demeure encore l'apanage des privilégiés. En créant le Musée européen des copies, Charles Blanc prétend rendre les collections européennes accessibles à tous. Blanc considère le chef-d'œuvre comme un élément fondamental de la formation de tout être humain. Il lui attribue un rôle essentiel dans la dignité de l'Homme. Nous pouvons lire dans La Grammaire des Arts du Dessin :

Quand je suis en présence d'un chef-d'œuvre, j'éprouve le besoin de mettre mon âme à l'unisson. Si j'avais le sentiment de mon indignité, l'admiration serait pour moi un malaise, un reproche; je me sentirais humilié de toute pensée basse: je ferais donc effort, une fois rentré dans moi-même, pour effacer de ma nature les taches qui me seraient apparues à cette vive lumière que projette la beauté19.

Cette approche explique l'intérêt de Blanc pour la création d'une institution qui place le modèle de la salle des chefs-d'œuvre au service de l'éducation des Français.

\section{La copie comme outil de conservation}

La collection du Musée européen des copies ne répond pas uniquement au projet de démocratiser l'art et son enseignement. Une autre préoccupation absorbe Charles Blanc : la conservation du souvenir des chefs-d'œuvre.

[En parlant du Musée européen des copies] Comment qu'on l'envisage, cette fondation était bonne. Non-seulement elle devait contribuer à instruire le peuple en lui montrant une image fidèle de chefs-d'œuvre dont il ne verra jamais les originaux, qui sont d'ailleurs fatalement condamnés à périr $[. . .]^{20}$.

Charles Blanc dresse un constat dramatique, quand il évoque la destinée des œuvres d'art du passé. Cette préoccupation est d'ailleurs très répandue à l'époque. Charles Clément souligne que cette fragilité touche tout particulièrement les fresques:

Il y a d'ailleurs une autre raison qui engagera l'administration à faire exécuter des reproductions aussi fidèles que possible de ces œuvres si belles et si importantes au point de vue de l'histoire de l'art. Ces fresques, qui datent de cinq ou six siècles, se détruisent de jour en jour. Un grand nombre d'entre elles ont déjà disparu, d'autres sont très gravement endommagées, et, pour ne citer qu'un exemple, c'est à peine si l'on voit encore quelques traces de la plupart des admirables peintures qui ornaient le Campo-Santo de Pise. Notre génération est peut-être la dernière qui pourra se faire une idée à peu près exacte, et par les originaux, de ce grand art décoratif dont nous voudrions voir un certain nombre d'échantillons dans le Musée européen ${ }^{21}$.

21 La question de la conservation des fresques est déjà ancienne. Grâce à l'ouvrage de Jacques Guillerme, L'atelier du temps ${ }^{22}$, nous pouvons constater que les problèmes de 
conservation des grands chefs-d'œuvre, et tout particulièrement des fresques, suscitent des débats depuis le $\mathrm{XVII}^{\mathrm{e}}$ siècle. Plusieurs projets visent à conserver le souvenir des œuvres en danger. La préoccupation principale est de découvrir une technique capable de rendre les images impérissables. En plus de la gravure, la mosaïque et l'émail ont parfois été choisis pour retranscrire les grandes compositions de l'histoire de l'art ${ }^{23}$.

En ce sens, le musée de Charles Blanc peut être compris comme la concrétisation d'une préoccupation de longue date. Il doit permettre de conserver le souvenir des chefsd'œuvre dans le cas où l'œuvre originale disparaît. Cette dimension du Musée européen des copies est particulièrement importante; elle soulève la possibilité de changer le statut d'une copie. En effet, si l'œuvre d'art originale disparaît, sa copie, comme reflet le plus exact de l'œuvre détruite, devient en quelque sorte presque un original.

La collection du Musée européen de Copies est donc appelée à devenir une collection de souvenirs, dont la visite sera indispensable pour tous ceux qui s'intéressent à l'histoire de l'art. Dans le cas où les chefs-d'œuvre reproduits viennent à disparaître, la collection du Musée européen change de statut et se rapproche ainsi de celle du Louvre, comme lieu de conservation de l'histoire de l'art.

\section{La relation avec le Salon : récupération du système d'émulation}

La relation entre le Musée européen des copies et le Salon n'est pas abordée dans la littérature secondaire. Pourtant, en lisant les écrits de Charles Blanc, on comprend que cette relation offre la clé qui permet de comprendre le dispositif mis en place au Palais de l'industrie en 1873.

Jusqu'en 1848, le Salon des artistes vivants a pris place au musée du Louvre. L'organisation de cette exposition est complexe, en raison du nombre d'œuvres à exposer, des rivalités entre les artistes mais, surtout, en raison de la mise en place de l'exposition au Louvre. Cependant, malgré la difficulté de l'entreprise, le dispositif proposait un système essentiel d'émulation entre les artistes modernes et les maîtres anciens. Le public devait mesurer l'avancement de l'art moderne dans le contexte d'une représentation du devenir de l'art.

Andrew McClellan, dans son ouvrage Inventing the Louvre ${ }^{24}$, va encore plus loin dans son analyse du dispositif. Selon lui, grâce à ce mode d'exposition, l'école française moderne est perçue comme la seule capable de succéder aux grandes écoles du passé. La peinture moderne française est ainsi rehaussée comme supérieure aux autres écoles nationales contemporaines ${ }^{25}$. Au vu de l'importance attribuée à ce système d'émulation, nous devons nous demander pourquoi il disparaît en 1848.

Dans les nombreux textes de Charles Blanc, nous trouvons une seule mention du Salon, mais comme nous le verrons, il s'agit d'un commentaire d'une grande importance :

La réunion de ces copies, exécutées dans les proportions mêmes de l'original, devait avoir ce double avantage: présenter au public le spectacle historique des œuvres d'art les plus belles qui soient en Europe, et rappeler aux artistes les exemples qu'ont laissés leurs ancêtres. Mais pour qu'une pareille exhibition portât ses fruits, il importait qu'elle se fit dans le palais même affecté aux expositions annuelles; il fallait que nos jeunes peintres eussent à subir le voisinage redoutable, sinon des grands maitres, au moins de leur image, ou si l'on veut de leur ombre ${ }^{26}$.

L'emplacement que Charles Blanc choisit pour le Musée européen des copies répond directement au but de cette nouvelle institution: instaurer à nouveau un système d'émulation entre les artistes contemporains et les anciens. En l'installant au Palais de 
l'industrie voisinant le Salon des artistes vivants, Charles Blanc propose de facto la récupération du dispositif en vigueur jusqu'à 1848 , quand le Salon s'ouvrait au Louvre.

Charles Blanc a sans doute décidé de récupérer un dispositif d'émulation qui met conjointement en scène les modernes et les anciens, en raison de son point de vue sur la situation contemporaine de la peinture, qu'il jugeait préoccupante. Il nous dit :

Voilà comment, sans entraver le moins du monde la liberté des artistes, sans même y toucher, l'État, en évoquant la tradition, peut exercer une influence salutaire sur les destinées de l'art, et en particulier sur les destinées de la grande peinture...

Il y a une grande peinture, en effet, qui n'est pas la même que la peinture grande, et ce n'est pas un des moindres travers de notre école, que d'avoir laissé se corrompre cette notion si élémentaire et si importante des convenances en matière de proportion. [...] En somme, la peinture en France est maintenant, de tous les arts, celui qui est le plus entaché d'hérésie, le plus dévoyé27.

Cette hypothèse semble d'autant plus plausible que Charles Blanc a décidé en 1872 de changer le règlement du Salon des artistes vivants. Dans son rapport au ministre de l'Instruction publique, publié dans La Chronique des arts et de la curiosité le 24 décembre 1871 , nous pouvons lire :

Il n'y a qu'une seule mesure efficace et qui réponde aux devoirs du gouvernement : c'est d'attribuer seulement le droit de suffrage aux artistes qui ont reçu le premier degré d'initiation, en obtenant soit le prix de Rome, soit une distinction marquée dans les expositions antérieures ${ }^{28}$.

En limitant considérablement le nombre des membres du jury et en les sélectionnant parmi les artistes dotés d'un prix de Rome, Charles Blanc peut imposer des critères de choix plus stricts, et plus fidèles à son idéal de la peinture. Le profil des membres appelés à siéger dans le jury du Salon coïncide parfaitement avec celui des artistes appelés à produire des copies pour le Musée européen.

En conclusion, la reprise du dispositif d'émulation qui met en scène le présent et le passé relève d'un choix parfaitement conscient, qui justifie entre autres l'emplacement du Musée européen des copies.

\section{Le problème de l'originalité}

La récupération de ce système d'émulation pose cependant un problème, qui touche à la relation tout à fait particulière que Charles Blanc entretient avec la notion d'original. En effet, dans une des citations que nous avons analysées auparavant, Blanc se montre conscient de la différence essentielle qui sépare une copie d'un original. Un peu plus loin nous pouvons lire les phrases suivantes :

De cette manière, les visiteurs d'un côté, de l'autre les artistes, en passant comme d'un temple à un bazar, auraient senti la différence. On aurait mesuré, on aurait peut-être essayé de franchir la distance qui nous sépare du temps où Raphaël décorait la Farnésine, où Michel-Ange faisait plafonner dans la chapelle du pape des figures sublimes qu'il avait vues, non pas traverser les rues de Rome, mais se peindre au fond de cette chambre obscure qui était son âme. Ainsi, sans aucune oppression, sans aucun pédantisme, rien qu'en laissant la parole aux peintres d'autrefois, on eût procuré à la jeunesse un enseignement élevé et profitable, une austère leçon ${ }^{29}$.

Charles Blanc n'utilise pas le mot copie. Il assimile les copies d'après les chefs-d'œuvre aux chefs-d'œuvre eux-mêmes. Il va même jusqu'à comparer le Musée des copies à un temple, terme couramment employé pour décrire les salles de chefs-d'œuvre ${ }^{30}$. La référence à l'âme de l'artiste est encore plus étonnante, car les détracteurs du projet 
critiquent précisément, dans les copies présentées au musée, l'absence de l'âme de l'artiste. Nous pouvons le lire dans un texte d'Henri Delaborde :

[...] si exacts qu'on les suppose, ces fac-similés d'anciens tableaux ne mériteront jamais notre entière confiance. Dans ce travail de seconde main, l'âme secrète, l'inspiration intime qui vivifiait l'œuvre originale se sera comme engourdie en raison même des efforts accomplis par le traducteur, et les patientes insistances de l'outil n'auront réussi qu'à rendre plus ou moins correctement les apparences, alors que l'essentiel eût été de nous révéler le fond des choses ${ }^{31}$.

En conclusion, Charles Blanc comprend l'idée d'originalité d'une manière ambiguë, qui continue de nous surprendre aujourd'hui. À son époque déjà, elle a suscité un grand nombre de critiques. Son appropriation, aussi bien du dispositif de la salle des chefsd'œuvre, que du dispositif d'émulation entre les artistes du passé et les artistes modernes, repose sur cette conception tout à fait personnelle de l'œuvre d'art originale.

Le décalage entre le point de vue de Blanc et celui plus généralement partagé est alors total. Les mots du critique Albert Wolff nous aideront à comprendre cette différence essentielle. Wolff imagine la réaction des grands maîtres du passé face aux copies de leurs œuvres exposées au Musée européen :

Velasquez s'arrachait les cheveux; Raphaël poussait les gémissements qui avaient attiré l'attention dudit passant. Le Titien, accroupi devant une copie de son œuvre, ne disait rien, mais son attitude trahissait sa douleur [...] - Messieurs et chers camarades, dit enfin une voix que le commissaire reconnut pour celle de MichelAnge, sous aucun gouvernement et à aucune époque de l'histoire, une administration quelconque des beaux-arts n'a ouvert de musée plus attristant que celui-ci. En présence de cet attentat contre la peinture que faut-il faire ? - Il faut voter pour Barodet afin de donner une leçon au gouvernement qui nous maltraite de la sorte, fit [...] Raphaël d'une voix douce et émue ${ }^{32}$.

Chez Wolff, la copie est entendue comme un outrage aux maîtres du passé. La comparaison des copies aux originaux est impossible car en réalité ces copies sont considérées comme des insultes au grand art de la peinture.

\section{La question de la peinture}

Le Musée européen des copies a été un échec retentissant. Cependant, il a été mené dans des conditions politiques difficiles. À nos yeux, le problème de la reproductibilité a motivé la dissolution du projet de Charles Blanc. Louis Fiaux, dans son ouvrage consacré à la figure de Charles Blanc, nous donne peut-être, sans en être conscient, la clé de l'échec du Musée européen. En plaidant en faveur du projet il nous dit :

Si la sensation éprouvée devant l'œuvre originale vous ressaisit à nouveau devant la copie, le but n'est-il point atteint? Pour la statue, le bas-relief, nul ne discute, chacun accepte le moulage, la reproduction de toute grandeur et l'homme de sens artistique se plaît jusque devant la statuette de la Vénus de Milo ou l'Athlète de Phidias. Qui critique la gravure, la photographie, multipliées à l'infini et mettant à la portée des petites fortunes les plus remarquables œuvres de l'art moderne et ancien. Dans cet ordre d'idées, n'était-elle point digne d'un homme public, la pensée de rassembler dans une ville telle que Paris et d'offrir à la foule [...] la copie en peinture des chefs-d'œuvre de Dresde, d'Amsterdam, de Florence ou de Rome ${ }^{33}$ ?

Louis Fiaux soulève ici la question de l'appréciation différenciée des copies de peinture, qui ne relèvent pas de la même catégorie que les moulages et les autres techniques de reproduction. Au XIX $x^{e}$ siècle, les techniques permettant de reproduire les œuvres d'art 
se multiplient. Cette nouvelle reproductibilité est bien acceptée quand il s'agit de sculptures, de gravures ou encore de photographies. La peinture, cependant, semble constituer la seule exception à cette nouvelle règle, en raison du procédé employé pour l'exécution des copies.

Nous l'avons vu à travers la citation de Louis Fiaux, la sculpture d'édition est une pratique parfaitement établie au $\mathrm{xIX}^{\mathrm{e}}$ siècle. La sculpture est considérée comme un art $\mathrm{du}$ multiple, dans lequel interviennent deux moments de production clairement dissociables, le moment de la composition ou inventio, et le moment de l'exécution ${ }^{34}$.

Le mode de production de la sculpture au xIX siècle implique l'intervention de plusieurs personnes dans l'exécution de l'œuvre finale. L'artiste apporte un modèle qui est retranscrit dans le marbre ou dans le bronze par un praticien ou un fondeur. Le sculpteur ne laisse pas sa trace directe sur le marbre, qui est en réalité une reproduction mécanique de l'objet sur lequel l'artiste a travaillé.

31 Cette distinction est propre à la sculpture et elle a permis, comme l'explique Florence Rionnet dans sa contribution au colloque De main de maitre : l'artiste et le faux ${ }^{35}$, d'exposer ensemble des sculptures originales et des copies. Les copies en plâtre, marbre ou bronze sont alors perçues comme un moyen légitime de transmettre l'idée des originaux. Ceci n'est possible que grâce au développement des nouvelles techniques de reproduction, et de réduction mécaniques.

Cependant, si nous essayons d'appliquer cette réalité à l'art de la peinture, nous constatons rapidement que la situation est tout autre. Dans le cas de la peinture, la production de l'œuvre et sa conception mentale sont indissociables. En conséquence, la reproduction d'un tableau est impossible. En peinture, copier revient à modifier. La copie de peinture n'est pas considérée comme une duplication de l'œuvre d'art originale, mais comme une réinterprétation de celle-ci.

\section{Le regard de Charles Blanc}

Le projet du Musée européen des copies repose sur une vision de la peinture, et par conséquent de la copie en peinture, bien différente de celle que nous venons d'évoquer. Charles Blanc scinde l'œuvre d'art en deux éléments: l'image idéale et l'œuvre matérielle. Ce point de vue est partagé à l'époque par des auteurs influents tel Victor Cousin. Dans son œuvre Du vrai, du beau et du bien, ce philosophe évoque les différents arts et explique à ce propos :

Il faut donc que l'artiste s'attache à représenter l'idéal. Tout a son idéal. Le premier soin de l'artiste sera donc, quoi qu'il fasse, de pénétrer d'abord l'idéal caché de son sujet, car ce sujet en a un, pour le rendre ensuite plus ou moins frappant aux sens et à l'âme, selon les conditions que lui imposent les matériaux même qu'il emploie, la pierre, la couleur, le son, la parole. [...] L'expression est essentiellement idéale : ce que l'expression tente de faire sentir, ce n'est pas ce que l'œil peut voir et la main toucher, c'est évidemment quelque chose d'invisible et d'impalpable ${ }^{36}$.

Cette distinction permet à Charles Blanc de considérer la copie en peinture comme une transcription fidèle de l'image idéale d'un original, malgré les différences de facture. Aux yeux de Blanc, les copies exposées au Musée européen des copies sont en mesure de transmettre aux visiteurs une caractéristique essentielle des originaux, leur image idéale. 
De ce point de vue, tout à fait particulier, la copie de peinture fournit l'outil adéquat pour mener à bien le projet de Charles Blanc. La collection du Musée européen des copies complète celle du Louvre, et propose ainsi un panorama complet de l'histoire de l'art à Paris. Elle conserve le souvenir des œuvres en péril ; elle permet de réinstaurer le système d'émulation des artistes anciens et modernes, établi entre le Louvre et le Salon des artistes vivants, et qui avait survécu jusqu'en 1848 .

\section{NOTES}

1. Charles Blanc, Grammaire des arts du dessin, préfacé et annoté par Claire Barbillon, Paris, École nationale supérieure des beaux-arts, 2000. Sur Blanc: article de Claire Barbillon dans le Dictionnaire des historiens de l'art (https://www.inha.fr/fr/ressources/publications/publicationsnumeriques/dictionnaire-critique-des-historiens-de-l-art.html).

2. Sur le Musée européen des copies : Albert Boime, "Le Musée des Copies ", Gazette des beauxarts, 6. Pér., 64 (1964), pp. 237-247. Pierre Vaisse : "Charles Blanc und das "Musée des Copies" ", Zeitschrift für Kunstgeschichte, 39 (1976), pp. 54-66. Duro, Paul : «Le Musée des copies de Charles Blanc à l'aube de la III République : catalogue ", Bulletin de la Société de l'histoire de l'art français, 1985, pp. 283-313. Paul Duro, “"Une impulsion vigoureuse aux travaux d'art..." : la création du Musée des Copies ", dans Les collections: fables et programmes, Jacques Guillerme (dir.), Seyssel, Éditions Champ Vallon, 1993, pp. 283-288. Robert Scherkl, «Charles Blancs Musée des copies: Kopien wie Originale? », Zeitschrift für Kunstgeschichte, 63 (2000), pp. 358-371.

3. Rapport de Charles Blanc à Jules Simon, 26 octobre 1871, Archives Nationales (AN) F21 572.

4. Rapport de Charles Blanc à Jules Simon, 26 octobre 1871, AN. F21 572.

5. Littérature secondaire: France Lechleiter, Les envois de Rome des pensionnaires peintres de l'Académie de France à Rome de 1863 à 1914, thèse de doctorat sous la direction de Bruno Foucart, université Paris IV Sorbonne, 2008, document disponible à l'adresse : http://www.theses.parissorbonne.fr/these.lechleiter.pdf.

6. Rapport de Charles Blanc à Jules Simon, 26 octobre 1871, AN. F21 572

7. Pierre Vaisse, La Troisième République et les peintres, Paris, Flammarion, 1995, pp. 168-171.

8. Paul Duro, "The "Demoiselles à Copier" in the Second Empire ", Woman's Art Journal, vol. 7, n 1 (Spring-Summer, 1986), pp. 1-7.

9. Charles Blanc, «L'état des Beaux-Arts en France à la veille du Salon de 1874. I peinture ", Le Temps, 7 avril 1874, [s. p.].

10. Louis Auvray, Le Musée européen : copies d'après les grands maitres, au palais des Champs-Élysées, Paris, Renouard, 1873.

11. Sur Philippe-Auguste Jeanron et sa réforme du musée du Louvre: Madeleine Rousseau et Marie-Martine Dubreuil, La vie et l'œuvre de Philippe-Auguste Jeanron : peintre, écrivain, directeur des musées nationaux, 1808-1877, Paris, Ed. de la Réunion des musées nationaux, 2000. Geneviève BrescBautier, Guillaume Fonkenell et Jacques Foucart, « Le musée moderne de Jeanron (1848-1851) », dans Geneviève Bresc-Bautier, Guillaume Fonkenell et Françoise Mardrus (dir.), Histoire du Louvre, Paris, Musée du Louvre, Fayard, 2016, tome II, pp. 105-157.

12. Dominique Lobstein, Les Salons au XIX siècle: Paris, capitale des arts, Paris, Éditions la Martinière, 2006, pp. 137-149.

13. M. Rousseau et M.-M. Dubreuil, op. cit. note 11. 
14. Cecilia Hurley, François Mairesse, "In the shadow of the Tribuna ", Studiolo, $n^{\circ} 9,2012$, pp. 128-140.

15. C. Hurley, F. Mairesse, op. cit. note 14, p. 135.

16. Hans Belting, Le chef-d'œuvre invisible, Nîmes, Éditions Jacqueline Chambon, 2003.

17. Charles Blanc, La collection d'objets d'art de M. Thiers léguée au Louvre, Paris, Jouaust et Sigaux, 1884, p. XII.

18. L. Auvray, op. cit. note 10, p. 12.

19. C. Blanc, op. cit. note 1, p. 48.

20. C. Blanc, op. cit. note 9, [s. p.].

21. Charles Clément, "Le Musée européen », Journal des Débats Politiques et Littéraires, 25 avril 1873 , p. 3.

22. Jacques Guillerme, L'atelier du temps. Essai sur l'altération des peintures, Paris, Hermann, 1964.

23. Idem, ibidem, pp. 118-122.

24. Andrew McClellan, Inventing the Louvre: art, politics, and the origins of the modern museum in Eighteenth-Century Paris, Berkeley, University of California Press, 1999.

25. Idem, ibidem, p.81.

26. C. Blanc, op. cit. note 9, [s. p.].

27. C. Blanc, op. cit. note 9, [s. p.].

28. Charles Blanc, «Rapport au ministre de l'instruction publique, des cultes et des beaux-arts, sur l'exposition nationale de 1872 suivi du règlement de l'exposition nationale des ouvrages des artistes vivants pour 1872 ", La Chronique des arts et de la curiosité, 24 décembre 1871, pp. $28,29$.

29. C. Blanc, op. cit. note 9, [s. p.].

30. C. Hurley, F. Mairesse, op. cit. note 14, p. 136.

31. Henri Delaborde, "Le Musée des Copies ", La Revue des deux mondes, tome 150, $43^{\mathrm{e}}$ année, seconde période, 1873, p. 209.

32. Albert Wolff, «Le musée des horreurs », Le Figaro, 18 avril 1873, p. 1.

33. Louis Fiaux, Charles Blanc, Paris, G. Marpon et E. Flammarion, 1882, p. 47.

34. Pamella Guerdat, "L'un et le multiple. De la conception à l'œuvre diffusée ", dans Pascal Griener et Pamella Guerdat (dir.), Marcello. Une sculptrice à l'œuvre. Correspondance I, Fribourg, Société d'histoire du canton de Fribourg, 2015, p. 157.

35. Actes du colloque De main de maître : l'artiste et le faux, Paris, musée du Louvre, publiés sous la direction de Clément Chéroux, Paris, Hazan, musée du Louvre, 2004.

36. Victor Cousin, Du vrai, du beau et du bien, Paris, Didier, 1862, pp. 189, 190.

\section{RÉSUMÉS}

Le présent article se propose d'analyser la construction idéologique du Musée européen des copies dans la relation d'appropriation avec le musée du Louvre. Le Musée européen des copies est relativement méconnu en raison de sa courte vie. En effet, ce projet imaginé principalement par l'historien de l'art et directeur des Beaux-Arts français Charles Blanc, fut inauguré en avril 1873 et resta ouvert à peine neuf mois. Charles Blanc imagina ce musée comme une institution qui devait s'articuler au musée du Louvre. La relation entre ces deux institutions est profonde et touche non seulement à la constitution de la collection, mais aussi à l'appropriation du modèle de la salle des chefs-d'œuvre mis en place au Louvre par Philippe-Auguste Jeanron en 1851 au Salon 
carré, ou encore à la récupération du système d'émulation entre les peintres anciens et modernes qui existait au Louvre jusqu'en 1848 quand le Salon des artistes vivants fut définitivement expulsé du musée parisien.

The present article proposes to analyse the ideological construction of the Musée européen des copies related to the appropriation with the Musée du Louvre. The Musée européen des copies is relatively little-known because of its short life. The project, mainly imagined by the French art historian and director of the École des beaux-arts Charles Blanc, was opened in April 1873 and remained open barely nine months. Charles Blanc conceived it as an institution that was to be attached to the Musée du Louvre. The relationship between the two institutions was profound and touched upon not only the constitution of the collection, but also the appropriation of the model of the room of masterpieces established in the Louvre by Philippe-Auguste Jeanron in 1851 at the Salon carré, and the recuperation of the system of emulation between ancient and modern painter that existed at the Louvre until 1848 when the Salon des artistes vivants was definitively evited from the Parisian museum.

\section{INDEX}

Keywords : Musée des copies, Charles Blanc, Louvre, Salon, masterpiece, copy, cast

Mots-clés : Musée des copies, Charles Blanc, Louvre, Salon, chef-d'œuvre, copie, moulage

\section{AUTEUR}

\section{ELISA RODRÍGUEZ CASTRESANA}

Elisa Rodríguez Castresana, actuellement assistante doctorante auprès du Prof. Pascal Griener à l'université de Neuchâtel, a réalisé une licence en histoire de l'art à l'université Complutense de Madrid où elle a commencé à s'intéresser à la muséologie et à l'étude des collections en lien avec l'histoire de l'art. Elle a confirmé ce choix en poursuivant ses études avec le master en études muséales proposé par l'université de Neuchâtel. Son mémoire portait sur le Musée européen des copies. Ce sujet passionnant donna naissance à son actuel projet de thèse, qui traite de l'étude du Musée européen des copies pour s'intéresser au phénomène de la copie au XIX siècle.

$* * *$

Elisa Rodríguez Castresana, currently a PhD student and assistant to Prof. Pascal Griener at the Université de Neuchâtel, obtained a BA in art history at the Universidad Complutense de Madrid where she became interested in museology and the study of collections in relation to art history. She confirmed this choice by pursuing her studies with the master's in museum studies proposed by the Université de Neuchâtel. Her dissertation was on the Musée européen des copies. This subject gave rise to her current thesis, which focuses on the study of the Musée européen des copies and the wider phenomenon of copying in the nineteenth century. 